\title{
Should the number of acute exacerbations in the previous year be used to guide treatments in COPD?
}

\author{
Mohsen Sadatsafavi (1) ${ }^{1,2,3}$, James McCormack ${ }^{2}$, John Petkau ${ }^{4}$, Larry D. Lynd ${ }^{2,5}$, \\ Tae Yoon Lee ${ }^{1,2}$ and Don D. Sin $^{3}$ \\ Affiliations: ${ }^{1}$ Respiratory Evaluation Sciences Program, Faculty of Pharmaceutical Sciences, University of \\ British Columbia, Vancouver, BC, Canada. ${ }^{2}$ Collaboration for Outcomes Research and Evaluation, Faculty of \\ Pharmaceutical Sciences, University of British Columbia, Vancouver, BC, Canada. ${ }^{3}$ UBC Centre for Heart Lung \\ Innovation and Dept of Medicine (Respirology), University of British Columbia, Vancouver, BC, Canada. ${ }^{4}$ Dept \\ of Statistics, University of British Columbia, Vancouver, BC, Canada. ${ }^{5}$ Centre for Health Evaluation and \\ Outcome Sciences, Providence Healthcare Research Institute, Vancouver, BC, Canada.
}

Correspondence: Mohsen Sadatsafavi, UBC Centre for Heart Lung Innovation and Dept of Medicine (Respirology), Faculty of Pharmaceutical Sciences, University of British Columbia, 7th Floor, 828 West 10th Avenue, Research Pavilion, Vancouver, BC, V5Z 1M9, Canada. E-mail: msafavidmail.ubc.ca

@ERSpublications

Dichotomisation of COPD exacerbation frequencies based on observed number of events in the previous year results in phenotypes that are inherently unstable, so much so that their suitability for informing treatment decisions should be seriously questioned https://bit.ly/34nFClc

Cite this article as: Sadatsafavi M, McCormack J, Petkau J, et al. Should the number of acute exacerbations in the previous year be used to guide treatments in COPD? Eur Respir J 2021; 57: 2002122 [https://doi.org/ $10.1183 / 13993003.02122-2020]$.

\section{ABSTRACT}

Background: In contemporary management of chronic obstructive pulmonary disease (COPD), the frequent exacerbator phenotype, based on a 12-month history of acute exacerbation of COPD (AECOPD), is a major determinant of therapeutic recommendations. However, there is considerable debate as to the stability of this phenotype over time.

Methods: We used fundamental principles in time-to-event analysis to demonstrate that variation in the frequent exacerbator phenotype has two major sources: variability in the underlying AECOPD rate and randomness in the occurrence of individual AECOPDs. We re-analysed data from two large cohorts, the Evaluation of COPD Longitudinally to Identify Predictive Surrogate Endpoints (ECLIPSE) study and the SubPopulations and InteRmediate OutcoMes In COPD Study (SPIROMICS), using a Bayesian model that separated these sources of variability. We then evaluated the stability of the frequent exacerbator phenotype based on these results. Results: In both cohorts, the pattern of AECOPDs strongly supported the presence of an individualspecific underlying AECOPD rate which is stable over time (Bayes Factor less than 0.001). Despite this, the observed AECOPD rate can vary markedly year-to-year within individual patients. For those with an underlying rate of $0.8-3.1$ events.year ${ }^{-1}$, the frequent exacerbator classification, based on the observed rate, changes more than $30 \%$ of the time over two consecutive years due to chance alone. This value increases to more than $45 \%$ for those with an underlying rate of $1.2-2.2$ events.year ${ }^{-1}$.

Conclusions: While the underlying AECOPD rate is a stable trait, the frequent exacerbator phenotype based on observed AECOPD patterns is unstable, so much so that its suitability for informing treatment decisions should be questioned. Whether evaluating AECOPD history over longer durations or using multivariate prediction models can result in more stable phenotyping needs to be evaluated.

This article has an editorial commentary: https://doi.org/10.1183/13993003.03758-2020

This article has supplementary material available from erj.ersjournals.com

All the data underlying this study are publicly available from the report of original studies. The collated data are provided in the supplementary material.

Received: 2 June 2020 | Accepted: 17 Aug 2020

CERS 2021. This version is distributed under the terms of the Creative Commons Attribution Non-Commercial Licence 4.0. 


\section{Introduction}

Chronic obstructive pulmonary disease (COPD) is a common disease of the airways responsible for a high proportion of hospitalisations and mortality in many parts of the world [1]. Acute exacerbations of COPD (AECOPDs) are a defining feature of the disease and a major source of morbidity and mortality [2]. Due to the high burden of AECOPDs, their prevention is a major target of current COPD management strategies. In several contemporary COPD guidelines, the observed AECOPD rate from the previous year is used to identify a frequent exacerbator phenotype, which in turn guides the choice of initial and subsequent treatments. For example, the Global Initiative for Chronic Obstructive Lung Disease (GOLD) recommends the use of inhaled corticosteroids (ICS) for patients who are "frequent exacerbators" based on a threshold of $\geqslant 2$ moderate AECOPDs or $\geqslant 1$ severe AECOPD in the previous 12 months [3]. The Canadian guidelines follow a similar definition for the frequent exacerbator phenotype [4].

Given its central role in informing therapeutic choices, the frequent exacerbator phenotype needs to be stable over time if it is to be used to provide logical and consistent treatment recommendations to patients. Several investigators have studied the stability of such phenotypes in longitudinal COPD cohorts [5-9]. Two highly cited examples are the Evaluation of COPD Longitudinally to Identify Predictive Surrogate Endpoints (ECLIPSE) study [5] and SubPopulations and InteRmediate OutcoMes in COPD Study (SPIROMICS) [6] cohorts. For each of these cohorts, the investigators used similar methods to determine the stability of the frequent and nonfrequent exacerbator phenotypes over 3 years. Interestingly, they arrived at different conclusions. The ECLIPSE investigators concluded that “...COPD with frequent exacerbation is a distinct phenotype..." and that the "...frequent exacerbation phenotype can be identified on the basis of a history of exacerbations...". On the other hand, the SPIROMICS investigators concluded that "...exacerbation frequency is highly variable over time..." and that "...frequent exacerbator status defined by $\geqslant 2$ exacerbations in every year is distinctly uncommon...”. Given these discordant findings, there is considerable debate on the importance (and even existence) of a frequent versus nonfrequent exacerbator phenotype in clinical practice and therefore its application in clinical practice guidelines [10].

In this work, we show that the variation in the observed number of AECOPDs in a given period is influenced by two major factors: the variability in the individual-specific, underlying AECOPD rate and the randomness in the occurrence of AECOPD events. Evaluating the pattern of AECOPDs from this perspective sheds light on the inconsistencies in the interpretations of previous studies and provides new insights into the utility of current phenotyping based on AECOPD history. The first objective of this study was to evaluate whether the data from the ECLIPSE and SPIROMICS studies support the presence of an individual-specific, underlying AECOPD rate which is stable over time. The second objective was to explore, based on the findings from the first objective, the randomness of observed AECOPD counts in a 12-month period, in order to determine the suitability of this factor for phenotypic classification.

\section{Methods}

\section{Individual-specific underlying AECOPD rate as the true characteristic}

In the analysis of recurrent events, two related but distinct concepts exist. One is the intensity function (also referred to as the instantaneous rate) of an event and the second is the actual frequency of events [11]. The intensity function for a given patient for a time interval captures the tendency of that individual to experience the event of interest at any given time within that interval. For example, given that more AECOPDs occur in the winter, the intensity function of AECOPDs has a higher value in the winter months than during the summer. The intensity function for an individual patient is not directly observable; however, it can be estimated when one observes AECOPDs in a sample of patients. The intensity function for recurrent events is analogous to the hazard function for non-repeated events (e.g. mortality).

For recurrent events such as AECOPD, during any time interval, the "expected" number of AECOPDs is equal to the area under the curve of the intensity function during this interval. However, for each individual patient, the "observed" number of events is a random variable. Fundamental theory in time-to-event analysis states that, under some general conditions, the number of events experienced during a period is a random variable that follows a Poisson distribution [12]. This is illustrated in a stylised example in figure 1a. Here, the intensity function of a hypothetical COPD patient is depicted. The underlying average event rate for this patient (the area under the curve) is 1.5 (note that while the actual number of events is always an integer number, the average event rate is a continuous variable that can take any non-negative value). That is, if we follow 10 patients with the same AECOPD intensity function for 12 months, they will experience, on average, 15 events in total. However, the number of AECOPDs for each of these patients is random.

For any given underlying AECOPD rate, the randomness of the observed number of AECOPDs can be studied using a Poisson distribution, as depicted in figure $1 \mathrm{~b}$ [13]. This panel demonstrates the probability 
a)

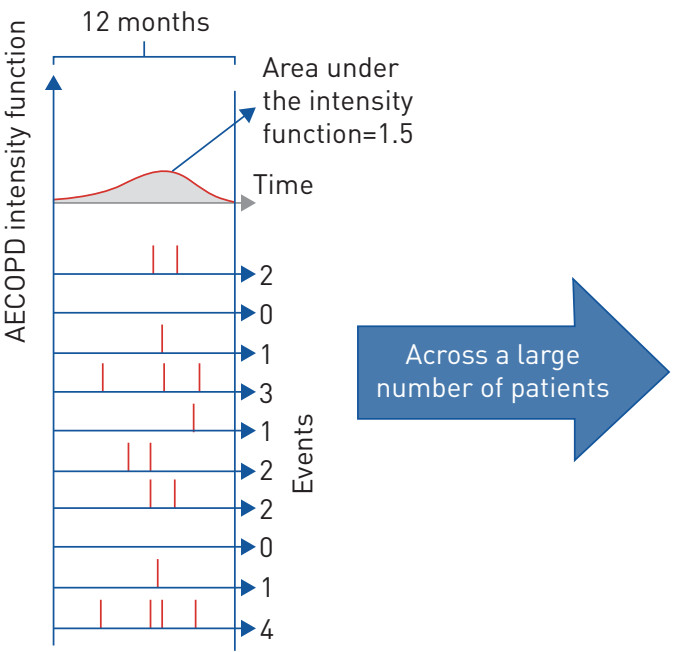

b)

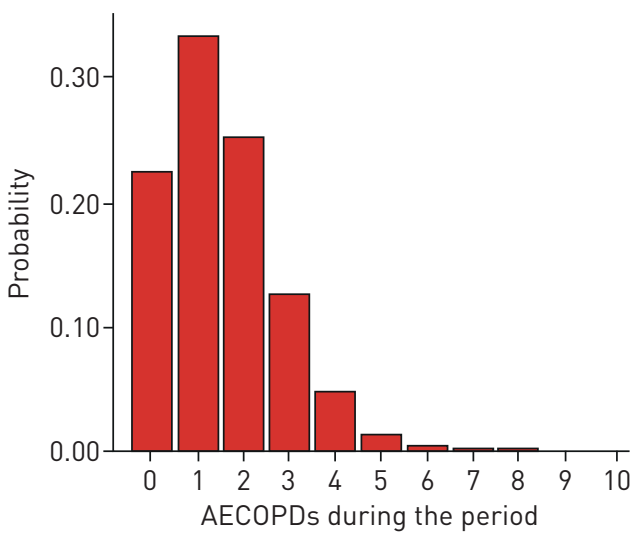

FIGURE 1 An illustrative example of the randomness of the observed number of acute exacerbations of COPD (AECOPDs). Panels are as follows: (a, top) the unobserved intensity function and (a, bottom) randomly generated event histories for 10 individuals. Vertical lines indicate the occurence of an event and, notably, the same intensity function results in a random number of events; (b) the number of events during any single period, which follows a Poisson distribution. COPD: chronic obstructive pulmonary disease.

of observing a certain number of AECOPDs during a 12-month interval for the same patient (with an underlying AECOPD rate of 1.5 per year). Had we known the underlying AECOPD rate for this patient, using a threshold definition of $<2$ AECOPDs.year ${ }^{-1}$, we would have classified this patient as a non-frequent exacerbator. However, according to figure 1, the patient only has a $22 \%$ chance of not experiencing any AECOPDs during a 12 -month period (the left-most bar) and a $34 \%$ chance of having exactly one AECOPD (the second bar). Thus, based on the observed AECOPD rate, this individual has only a $56 \%$ chance $(22 \%+34 \%)$ of being classified as a non-frequent exacerbator and a corresponding $44 \%$ chance of being incorrectly classified as a frequent exacerbator in any given 12-month period.

In this framework, the variation of the frequent-exacerbator definition over time can be decomposed into two components: the variation due to the potential instability of the underlying AECOPD rate over time and the randomness of observed AECOPD counts given the same underlying rate. These sources of variation can be formally studied using longitudinal AECOPD data.

\section{First objective: examining the presence of an underlying, stable AECOPD rate}

We re-analysed the observed AECOPD patterns in ECLIPSE and SPIROMICS to determine if the underlying AECOPD rate remains stable over time. ECLIPSE and SPIROMICS were two independent, non-interventional, multi-centre, 3-year prospective cohort studies, whose design is published elsewhere $[14,15]$. The overall objective of ECLIPSE was to evaluate COPD subtypes and factors that predict disease progression, while SPIROMICS aimed to identify distinct subgroups of COPD patients for targeted enrolment in future clinical trials and to discover and validate intermediate endpoints. Both studies pre-specified multiple endpoints, with AECOPDs as a specific outcome that was evaluated during each of the follow-up visits.

Our analyses are based on summary results from the two studies that evaluated the stability of the frequent exacerbator phenotype. The study based on the ECLIPSE cohort included 2138 patients [5], while that based on SPIROMICS included 1105 patients [6]. The demographic characteristics of these samples are provided in their respective publications $[5,6]$. Both studies classified each patient-year as having zero, one, or two or more (coded as two) AECOPDs. Over 3 years of observation, a patient would fall into one of 27 possible AECOPD count patterns (0/1/1,2/2/0, 1/0/1, etc). We used the reported number of patients within each of these 27 groups (obtained from the original publications) to fit two Bayesian hierarchical models. First, we fitted an "unstable underlying rate" model, which assumed that the underlying AECOPD rate varies randomly from one year to another and that individual patients do not differ systematically from each other in their underlying AECOPD rates. The second model was based on a "stable underlying rate" assumption, which assumed that each individual has a unique underlying AECOPD rate that remains constant over the 3-year span. This model separates between-individual variability (heterogeneity) in underlying AECOPD rate from chance variability in observed frequency. In both, the observed number of 
AECOPDs in a given year followed a Poisson distribution. In line with the original analyses, we did not adjust for any other variables. The models, implemented in OpenBUGS [16], are described in detail in the supplementary material.

After fitting these models, we determined to what extent they explained the observed AECOPD patterns in the two studies by plotting the observed frequencies for each of the 27 patterns against the corresponding predicted frequencies. We also calculated the Bayes factor to evaluate the amount of support in favour of the unstable underlying rate model versus the stable underlying rate model (the lower the Bayes factor, the higher the support for the stable model) [17].

\section{Second objective: examining the extent of variability of the frequent exacerbator phenotype}

As mentioned earlier, the observed number of AECOPDs will vary from year to year even in the presence of a stable underlying AECOPD rate. The utility of the current definition of the frequent exacerbator phenotype depends on the extent to which it remains stable from one year to another, such that AECOPD patterns from the previous year can inform clinicians to either step up or down therapies over time. Such stability can be quantified by calculating the probability that the frequent exacerbator classification of an individual with a given underlying AECOPD rate would randomly change in two consecutive years. This probability was calculated using the probability mass function of the Poisson distribution and was plotted as a function of the underlying AECOPD rate. To remain aligned with the original analyses of ECLIPSE and SPIROMICS, we used the definition of $\geqslant 2$ moderate/severe AECOPDs for the frequent exacerbator phenotype. Alternative definitions, including those adopted by GOLD and the American Thoracic Society (ATS) [18] are explored in the supplementary material.

\section{Results}

Is the underlying AECOPD rate stable in ECLIPSE and SPIROMICS?

The observed 3-year AECOPD patterns for both cohorts, as well as the predicted patterns for the unstable and stable underlying rate models, are given in figure 2 . We found that the unstable underlying rate model resulted in predicted relative frequencies for AECOPD counts that were widely different from the relative frequencies reported in both ECLIPSE and SPIROMICS. On the other hand, estimates were much closer to the observed relative frequencies in both cohorts for the stable underlying rate model. The average discrepancy (i.e. the mean absolute difference between the observed and predicted proportion of patients in each of the 27 categories) was $83 \%$ smaller for the stable underlying rate model compared with the unstable model in ECLIPSE. The corresponding value was $63 \%$ in SPIROMICS. The Bayes factor was $<0.001$, indicating substantial support for the stable underlying rate model. As such, data from ECLIPSE and SPIROMICS both support the presence of an individual-specific, stable underlying AECOPD rate, with the observed number of AECOPDs being a random variable with Poisson distribution.

\section{Why did ECLIPSE and SPIROMICS arrive at different conclusions regarding the stability of the frequent exacerbator phenotype?}

Given the above observation that the stable underlying rate model provides a very good explanation of the data for both studies, an obvious question is "...Why did the ECLIPSE and SPIROMICS investigators arrive at such discordant conclusions regarding the stability of the frequent exacerbator phenotype?...”. The answer lies in the distribution of the underlying AECOPD rates. As shown in figure 3, the distribution of the underlying AECOPD rates across patients was much more heterogeneous in ECLIPSE compared with SPIROMICS, with the former having a long tail indicating the presence of individuals who tended to exacerbate very frequently. The model-estimated proportion of frequent exacerbators (patients with an underlying AECOPD rate $\geqslant 2$ ) was $18.7 \%$ in ECLIPSE but only $3.5 \%$ in SPIROMICS. Thus, there was a scarcity of individuals in the true frequent exacerbator group in SPIROMICS. These differences in the distribution of underlying AECOPD rates between the two studies likely reflected different design strategies (i.e. individuals with mild GOLD grade I were not included in ECLIPSE). This is supported empirically by the first-year average annualised AECOPD rate per person, which was 0.38 in SPIROMICS and 1.21 in ECLIPSE.

\footnotetext{
Is the frequent exacerbator phenotype stable?

Given the findings from ECLIPSE and SPIROMICS, the results in this section are based on the assumption that the underlying AECOPD rate remains the same over two consecutive years and that the "noise" in the frequent-exacerbator phenotype is purely due to the randomness of AECOPD events. The probability of the patient being classified into different exacerbator categories over two consecutive years as a function of the underlying AECOPD rate, when the underlying AECOPD rate remains constant across the 2 years, is demonstrated in figure 4. This graph demonstrates that for those with an underlying AECOPD rate of between 0.8 and 3.1 events.year ${ }^{-1}$, the frequent exacerbator classification (defined by
} 

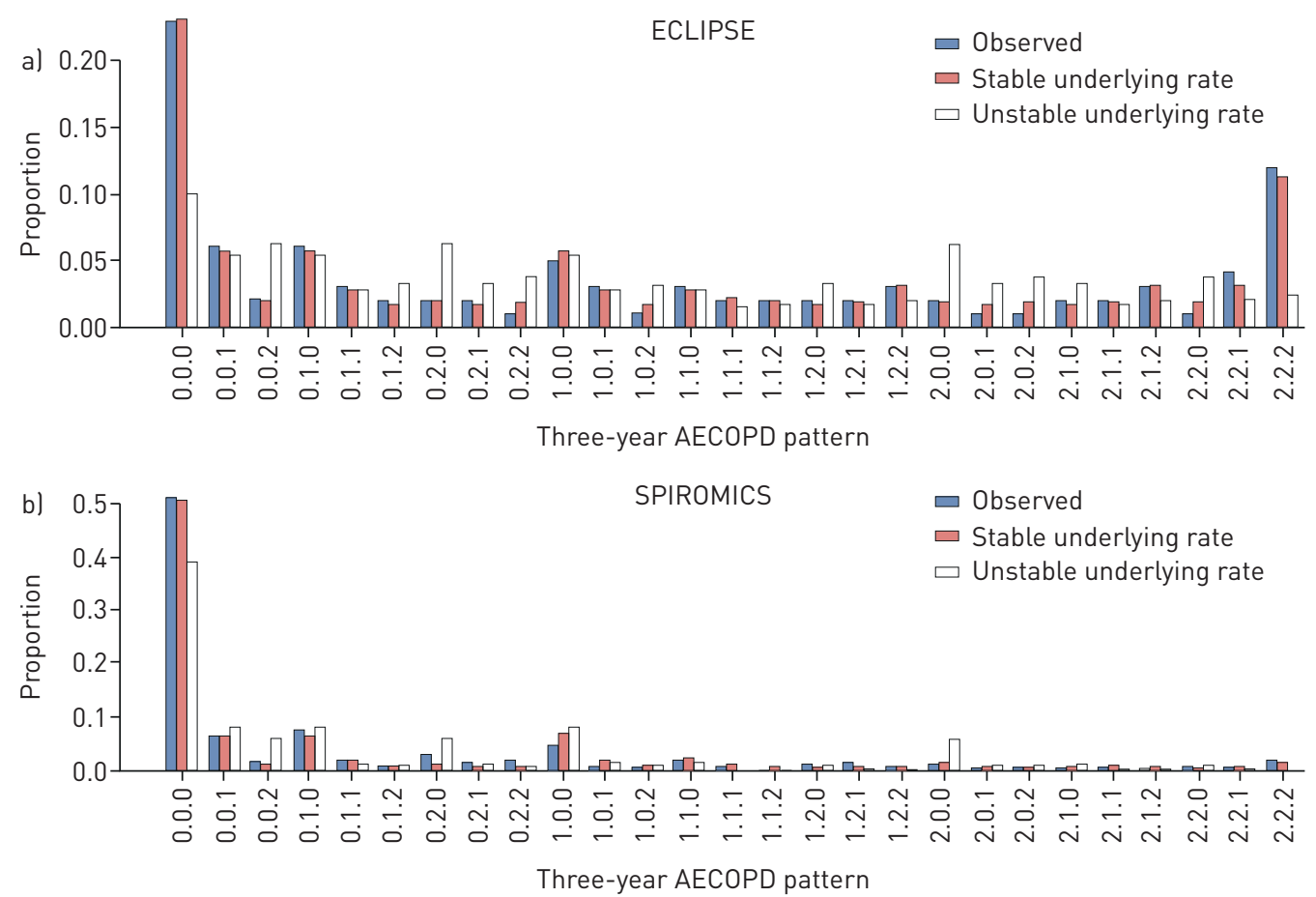

FIGURE 2 The observed versus the predicted proportion of patients across 27 3-year acute exacerbation of COPD (AECOPD) patterns in (a) the Evaluation of COPD Longitudinally to Identify Predictive Surrogate Endpoints (ECLIPSE) study and (b) the SubPopulations and InteRmediate OutcoMes In COPD Study (SPIROMICS). The labels on the $\mathrm{x}$-axis refer to the 3 -year pattern of AECOPDs le.g. 0.0 .0 refers to the proportion of patients who had zero AECOPDs in each of the 3 years, while 1.2.0 refers to the proportion of patients who had one AECOPD in year 1, two or more AECOPDs in year 2 and no AECOPDs in year 3). The mean absolute difference between observed frequency and predicted frequency was as follows: 0.022 (ECLIPSE: unstable underlying rate), 0.004 (ECLIPSE: stable underlying rate), 0.015 (SPIROMICS: unstable underlying rate) and 0.006 (SPIROMICS: stable underlying rate). COPD: chronic obstructive pulmonary disease.

using a categorisation of $\geqslant 2$ observed AECOPDs) changes more than $30 \%$ of the time from one year to the next. This probability increases to more than $45 \%$ for those with an underlying AECOPD rate of between 1.2 and 2.2 events.year ${ }^{-1}$. Again, this occurs despite the fact that the underlying AECOPD rate is assumed to be constant over the 2 years.

\section{Discussion}

One conundrum in the literature has been the stability (or instability) of the frequent exacerbator phenotype. Two influential studies (ECLIPSE and SPIROMICS) arrived at different conclusions in this

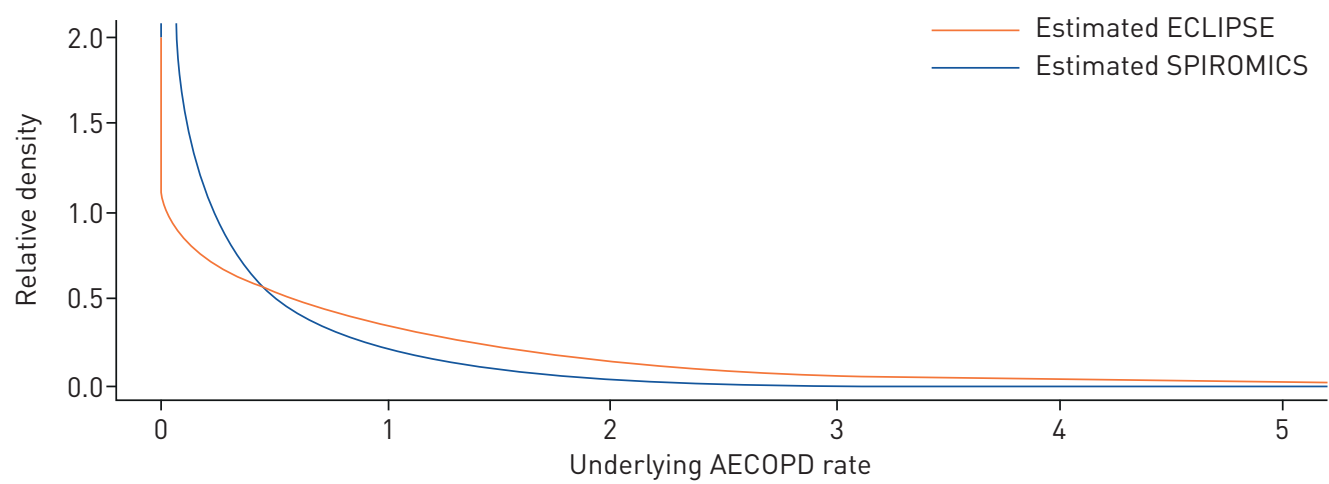

FIGURE 3 Estimated underlying acute exacerbation of COPD (AECOPD) rates for the Evaluation of COPD Longitudinally to Identify Predictive Surrogate Endpoints (ECLIPSE) study and the SubPopulations and InteRmediate OutcoMes In COPD Study (SPIROMICS) cohorts. COPD: chronic obstructive pulmonary disease. 


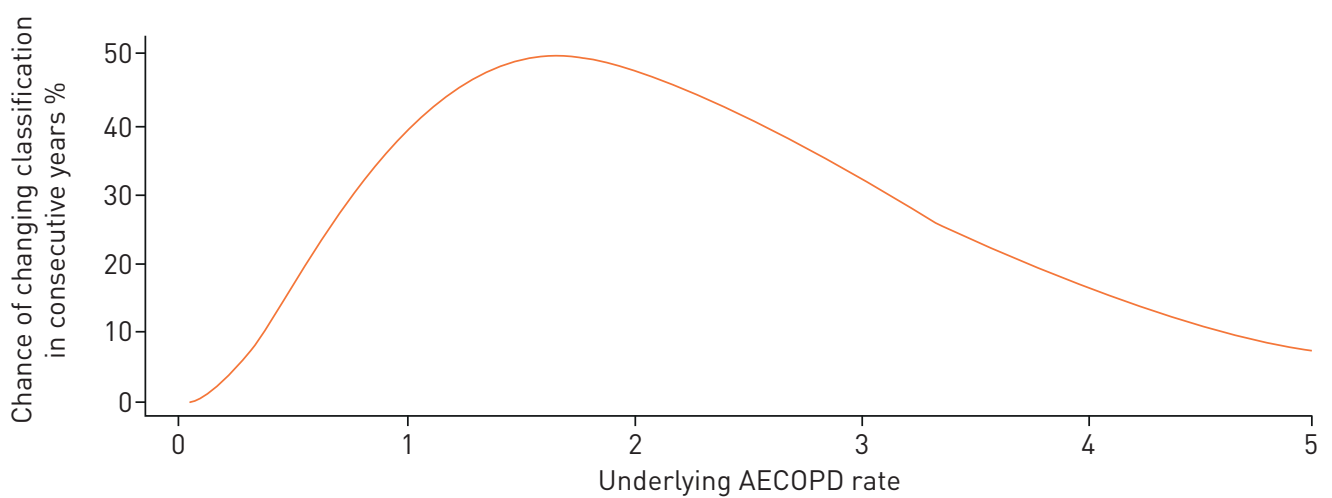

FIGURE 4 Probability of being classified into different exacerbator phenotypes over two consecutive years given a stable underlying acute exacerbation of COPD (AECOPD) rate. In line with the analysis of the Evaluation of COPD Longitudinally to Identify Predictive Surrogate Endpoints (ECLIPSE) study and SubPopulations and InteRmediate OutcoMes In COPD Study (SPIROMICS) cohorts, a frequent exacerbator is defined as having $\geqslant 2$ moderate/severe observed AECOPDs. For the curves that pertain to the Global Initiative for Chronic Obstructive Lung Disease (GOLD) definition ( $\geqslant 2$ moderate AECOPDs or $\geqslant 1$ severe AECOPD) or to the American Thoracic Society (ATS) definition ( $\geqslant 1$ moderate/severe AECOPD), please refer to the supplementary material. COPD: chronic obstructive pulmonary disease.

regard (one considering the phenotype to be stable, the other considering it to be unstable). In this work, we showed that the stability of the phenotype is affected by two major factors: the stability of the underlying AECOPD rate and the randomness in the occurrence of individual events. By re-analysing data from these two cohorts, we showed that both studies provide much stronger support for the stable model than the unstable model. Thus, the discordant interpretations of ECLIPSE versus SPIROMICS are likely due to important differences in the case mix between the two cohorts, rather than a difference in the instability of the underlying traits.

Despite the evidence supporting the presence of stable underlying AECOPD rates, we showed that the observed AECOPD rate in a 12 -month window can vary widely from year to year owing to the inherent randomness in the occurrence of AECOPDs. For individuals with an underlying AECOPD rate of between 0.8 and 3.1 events.year ${ }^{-1}$, the frequent exacerbation phenotype (based on the observed AECOPD rate) will change from one year to the next $30 \%$ or more of the time due to chance alone. A majority of patients with COPD harbour an underlying AECOPD rate in this range [19]. For patients whose underlying AECOPD rates are between 1.2 and 2.2 events.year ${ }^{-1}$, this probability increases to more than $45 \%$, which is close to the chance finding related to flipping a coin. Given the instability of the observed AECOPD rate from year to year, treatment recommendations based on an assessment of AECOPDs in the previous year may be problematic. This problem is exaggerated for pharmacotherapies that have only a modest effect in reducing AECOPD risk. Indeed, in such cases, any biological impact of the drug may be overshadowed by the large variability in AECOPD occurrence from year to year.

In addition to ECLIPSE and SPIROMICS, other studies have evaluated the stability of the frequent exacerbator phenotype. Using claims data from multiple sources, BLAGEV et al. [7] have determined the stability of severe AECOPDs over 6 years. They concluded that while the no-exacerbator phenotype was stable, the frequent exacerbator phenotype varied significantly over time. These findings can be explained by the fact that severe AECOPDs are much less frequent than moderate and severe ones. Thus, in a given year, most patients would not experience any severe exacerbations and would fall into the no-exacerbator phenotype. Only a small minority would experience multiple severe exacerbations per year (most likely by chance), with most of these patients experiencing no severe exacerbations in a subsequent year (akin to the phenomenon of regression to the mean) and resulting in an unstable frequent exacerbator phenotype. These results highlight the randomness of individual AECOPD events even when the underlying AECOPD rate is stable.

To ensure that our findings are interpretable in comparison with those of the original analyses of ECLIPSE and SPIROMICS, we used the same definition of frequent exacerbator and categorisation of 3 -year AECOPD patterns. For this same reason, we did not adjust for covariates. Given that the frequent exacerbator phenotype is defined purely based on the number of AECOPDs, the contribution of other factors (such as comorbidity) is not relevant for studying its stability. On the other hand, the effect of treatment on AECOPD rate deserves further consideration. Any time-varying exogenous factor that affects the risk of AECOPD, such as change in treatment, would be expected to act as an added source of variability in the underlying AECOPD rate. As such, one can argue that ECLIPSE and SPIROMICS data 
are even more compatible with the stable underlying rate model, given the potentially varying treatment in these cohorts.

To inform treatment decisions for AECOPD prevention, we need a phenotype definition that is more stable and less prone to the whims of chance. How can this be achieved? One possible solution may be to rely on information collected over longer periods (e.g. 3-4 years) for establishing AECOPD phenotypes, as previously suggested [9]. However, this approach would have limitations. For one, it would be impossible to apply this approach to recently diagnosed patients and there may be issues with patient recall of AECOPD events over a longer time horizon (although the latter may be addressed through more objective methods such as the use of electronic medical records). A more promising solution is to keep the assessment window to 12 months but to collect more information that may more clearly delineate the underlying AECOPD phenotypes. This can be achieved through the use of nuanced clinical prediction models that rely not just on AECOPD history but on a multitude of other predictor variables. A recent risk prediction model for AECOPD has demonstrated that the use of easily verifiable patient characteristics can significantly improve the predictability of AECOPDs, especially severe ones (an improvement in c-statistics of 0.11 was observed in this study) [20]. With the availability of multidimensional data, one can move beyond easily verifiable predictors to include values from imaging (e.g. bronchiectasis), blood or sputum biomarkers (e.g. eosinophils), comorbidity from electronic charts and other factors. In theory, the predicted risks from such models should be more stable over time because of the contribution of multiple patient characteristics that are relatively robust and less susceptible to the whims of chance. The effect of using multivariable prediction models to complement the influence of AECOPD history remains to be studied.

In summary, the current AECOPD phenotype definition is one that is informed by the conventional wisdom that "...the best predictor of future AECOPDs is previous AECOPD history..." [5]. However, as our results demonstrate, AECOPD history alone, while likely being the single best predictor for future AECOPDs, is not good enough to act as the basis for a reliable phenotype definition to inform treatment choices. To ascertain a more stable exacerbation phenotype we will likely need to move from the current parsimonious approach to a more comprehensive risk classification, one based on multiple patient and disease characteristics. The increasing availability of data-rich, longitudinal COPD cohorts, combined with the use of statistical methods that can separate true underlying patterns from chance variability, should enable the field to achieve this goal, which in turn will lead to more robust and consistent patient management strategies.

Author contributions: M. Sadatsafavi, J. McCormack, L.D. Lynd and D.D. Sin came up with the original idea. M. Sadatsafavi and J. Petkau formulated the research question. M. Sadatsafavi and T.Y. Lee performed the literature search. M. Sadatsafavi, L.D. Lynd and D.D. Sin designed the study. M. Sadatsafavi and J. Petkau performed the analyses. T.Y. Lee and J. Petkau reviewed the results and provided critical feedback. M. Sadatsafavi and D.D. Sin wrote the first draft. All authors provided substantial input for the manuscript.

Conflict of interest: M. Sadatsafavi has nothing to disclose. J. McCormack has nothing to disclose. J. Petkau has nothing to disclose. L.D. Lynd has nothing to disclose. T.Y. Lee has nothing to disclose. D.D. Sin reports grants and personal fees for lectures from AstraZeneca, personal fees for lectures from Boehringer Ingelheim, and grants from IKOMED, outside the submitted work.

Support statement: This study was funded by the Canadian Institutes of Health Research (catalyst grant 155554). The funder did not have any role in any aspects of this study and is not aware of this manuscript. Funding information for this article has been deposited with the Crossref Funder Registry.

\section{References}

1 Ehteshami-Afshar S, FitzGerald JM, Doyle-Waters MM, et al. The global economic burden of asthma and chronic obstructive pulmonary disease. Int J Tuberc Lung Dis 2016; 20: 11-23.

2 Kim V, Aaron SD. What is a COPD exacerbation? Current definitions, pitfalls, challenges and opportunities for improvement. Eur Respir J 2018; 52: 1801261.

3 Singh D, Agusti A, Anzueto A, et al. Global strategy for the diagnosis, management, and prevention of chronic obstructive lung disease: the GOLD science committee report 2019. Eur Respir J 2019; 53: 1900164.

4 Bourbeau J, Bhutani M, Hernandez $\mathrm{P}$, et al. Canadian Thoracic Society clinical practice guideline on pharmacotherapy in patients with COPD - 2019 update of evidence. Can J Respir Crit Care Sleep Med 2019; 3: $210-232$.

5 Hurst JR, Vestbo J, Anzueto A, et al. Susceptibility to exacerbation in chronic obstructive pulmonary disease. N Engl J Med 2010; 363: 1128-1138.

6 Han MK, Quibrera PM, Carretta EE, et al. Frequency of exacerbations in patients with chronic obstructive pulmonary disease: an analysis of the SPIROMICS cohort. Lancet Respir Med 2017; 5: 619-626.

7 Blagev DP, Collingridge DS, Rea S, et al. Stability of frequency of severe chronic obstructive pulmonary disease exacerbations and health care utilization in clinical populations. Chronic Obstr Pulm Dis 2018; 5: 208-220. 
8 Rothnie KJ, Müllerová H, Smeeth L, et al. Natural history of chronic obstructive pulmonary disease exacerbations in a general practice-based population with chronic obstructive pulmonary disease. Am J Respir Crit Care Med 2018; 198: 464-471.

9 Le Rouzic $\mathrm{O}$, Roche $\mathrm{N}$, Cortot $\mathrm{AB}$, et al. Defining the "frequent exacerbator" phenotype in COPD: a hypothesis-free approach. Chest 2018; 153: 1106-1115.

10 Lopez-Campos JL, Carrasco Hernández L, Muñoz X, et al. Current controversies in the stepping up and stepping down of inhaled therapies for COPD at the patient level. Respirol 2018; 23: 818-827.

11 Cook RJ, Lawless JF. The statistical analysis of recurrent events. New York, Springer, 2007.

12 Lawless JF. Regression methods for Poisson Process data. J Am Stat Assoc 1987; 82: 808-815.

13 Keene ON, Calverley PMA, Jones PW, et al. Statistical analysis of exacerbation rates in COPD: TRISTAN and ISOLDE revisited. Eur Respir J 2008; 32: 17-24.

14 Vestbo J, Anderson W, Coxson HO, et al. Evaluation of COPD longitudinally to identify predictive surrogate end-points (ECLIPSE). Eur Respir J 2008; 31: 869-873.

15 Couper D, LaVange LM, Han M, et al. Design of the subpopulations and intermediate outcomes in COPD study (SPIROMICS). Thorax 2014; 69: 491-494.

16 Lunn D, Thomas A, Best N, et al. WinBUGS - a Bayesian modelling framework: concepts, structure, and extensibility. Stat Comput 2000; 10: 325-337.

17 Hoijtink H, Mulder J, van Lissa C, et al. A tutorial on testing hypotheses using the Bayes factor. Psychol Methods 2019; 24: 539-556

18 Nici L, Mammen MJ, Charbek E, et al. Pharmacologic management of chronic obstructive pulmonary disease: an official American Thoracic Society clinical practice guideline. Am J Respir Crit Care Med 2020; 201: e56-e69.

19 Hoogendoorn M, Feenstra T, Hoogenveen R, et al. Association between lung function and exacerbation frequency in patients with COPD. Int J Chron Obstruct Pulmon Dis 2010; 5: 435-444.

20 Adibi A, Sin DD, Safari A, et al. The acute COPD exacerbation prediction tool (ACCEPT): a modelling study. Lancet Respir Med 2020; 8: 1013-1021. 